\title{
Ag nanoparticles sensitize IR-induced killing of cancer cells
}

Cell Research (2009) 19:1031-1034. doi: 10.1038/cr.2009.89; published online 21 July 2009

\section{Dear Editor,}

Nanosized particulate systems combining better cancer diagnosis with therapeutic effect are being designed based on the merging of nanotechnology with cellular and molecular techniques. The surface of these nanoscale carriers is often functionalized with biological molecules for stabilization and targeted delivery. The combinations of nano-core and associated functional molecules can cross the cell membrane [1], and the surface of nanomaterials (including coating and associated functional molecules) plays a critical role in determining the outcome of their interactions with cells $[2,3]$. Studying the potential effects of nanomaterials in biological systems often requires the administration of nanoparticles into a cell culture system or into living organisms in vivo. It should be noted, however, that under such conditions nanopaticles are known to adsorb proteins from the biological system, and the resulting heterogeneity in surface proteins may in turn affect the biological effects of nanomaterials [4, 5]. To minimize such non-specific effect, in this study we have modified the surface of our nanomaterials with proteins from fetal bovine serum (FBS), a supplement normally used for cell culture. Interestingly, we have recently found that for silane-coated monodisperse magnetite nanoparticles (MNPs), additional surface modification by FBS appears to facilitate particle uptake by cancer cells.

A number of previous studies have examined cytotoxicity elicited by nanoparticles after their uptake into cells under regular cell culture conditions [6-8]. However, the property of intracellular nanoparticles and their effect on cellular processes under more complex scenarios such as when cells are treated with ionizing radiation (IR) remain largely unclear. Recently, it was reported that certain gold nanoparticles (coated with polyethylene glycol (PEG) and amino acids) could enhance radiationinduced cytotoxicity in mice colorectal adenocarcinoma and breast cancer cells $[9,10]$. However, there has been a lack of systematic analyses on how different nanomaterials generally affect cellular radiation sensitivity, as well as the respective contributions by nanoparticles and their coatings. To address this issue, we have examined in this study three different nanomaterials, magnetite, gold and silver nanoparticles, with either distinct surface coatings (for magnetite paticles) or distinct size ranges (for gold and silver particles) (Supplementary information, Figure $\mathrm{S} 1$ ), for their potential effects on radiation-induced killing of glioma cells (experimental details in Supplementary information, Data S1). We chose glioma cells as a model system because most of glioblastoma multiform (GBM) is insensitive or even resistant to IR therapy, which is also reflected in the established glioma cell lines. Thus, enhancing the effect of IR-induced killing of glioma cells may offer therapeutic benefit for patients with GBM.

We prepared 12-nm monodisperse MNPs (Supplementary information, Figure S2), and coated them with one of the following four surface molecules: meso-2, 3-dimercaptosuccinic acid (DMSA), and silanes bearing various functional groups including amino group $\left(\mathrm{NH}_{2}\right)$, short-chain PEG, and carboxylic group (COOH) (Figure 1A). We also prepared gold nanoparticles (AuNPs) of diameter 10, 20 and $40 \mathrm{~nm}$, and silver nanoparticles (AgNPs) of diameter 20, 50 and $100 \mathrm{~nm}$ (Figure 1A). All the particles were modified with FBS followed by radiation-mediated sterilization (see Methods for details and Supplementary information, Figure S3, S4). We then examined the effects of these prepared nanoparticles on cell survival and cell radiation sensitivity in three different glioma cell lines, namely C6 (originated from mouse), U251 and SHG-44 cells (originated from human GBM).

We treated C6 glioma cells with a fixed concentration of MNPs $(0.1 \mathrm{mg} / \mathrm{ml})$, and compared the effects of different coatings. We found that DMSA- and PEG-silanecoated MNPs had little effect on glioma cell survival or cell sensitivity to radiation (Figure 1B). In contrast, treatment with $\mathrm{COOH}$ - and $\mathrm{NH}_{2}$-silane-MNPs led to a decrease of cell survival as indicated by the clonogenic assay (Figure 1B). Treatment with 6 Gy (gray, J/kg) of IR killed a significant portion of glioma cells, and the com- 
A

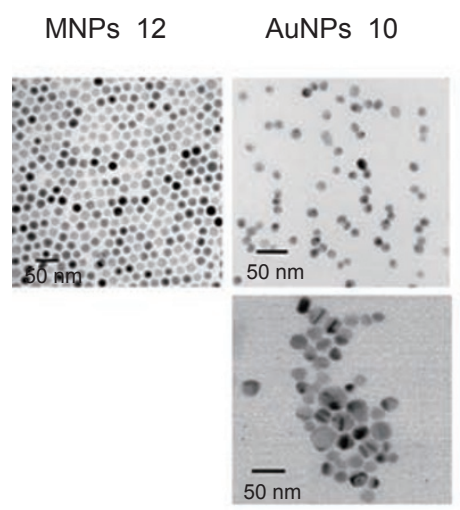

AgNPs 20

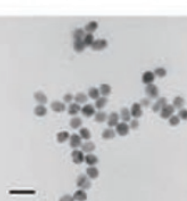

$50 \mathrm{~nm}$

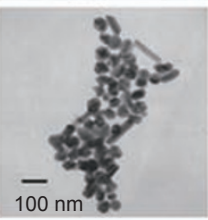

AgNPs 50
B

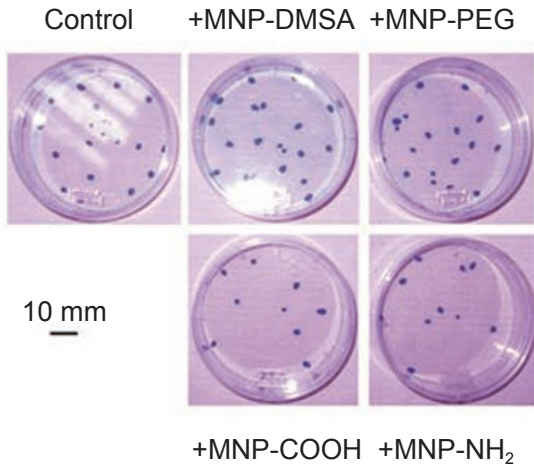

C

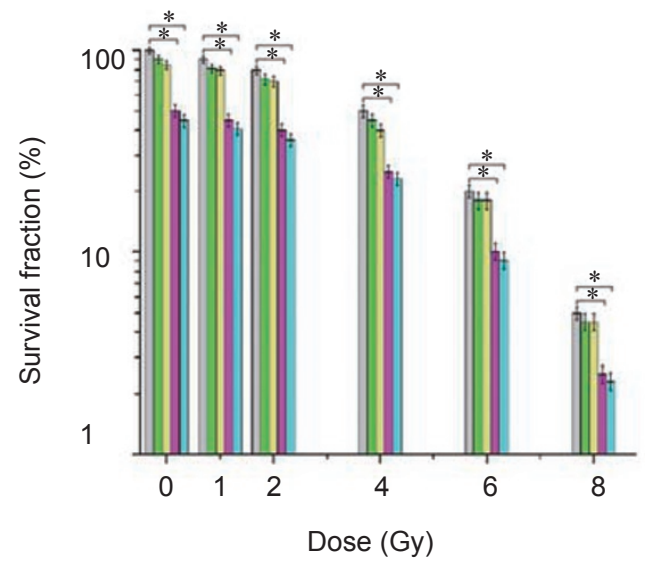

E

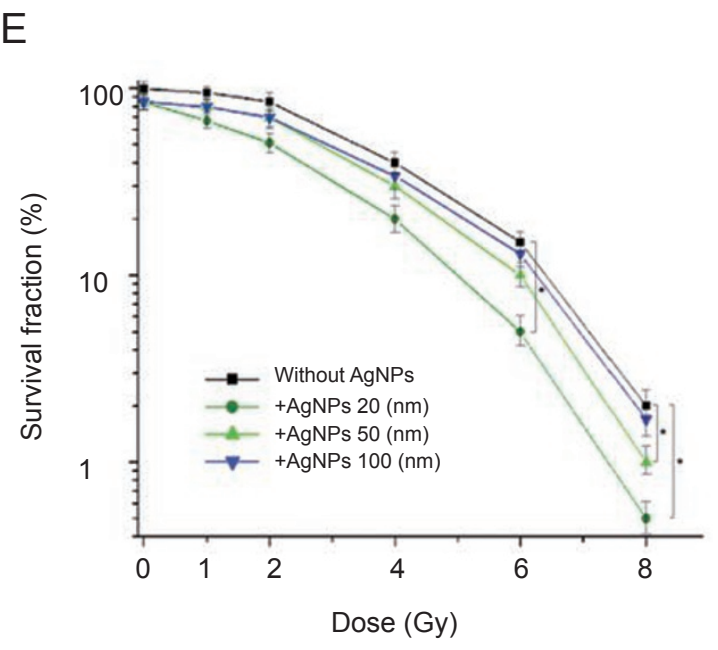

C

E
AuNPs $50 \quad(n m)$

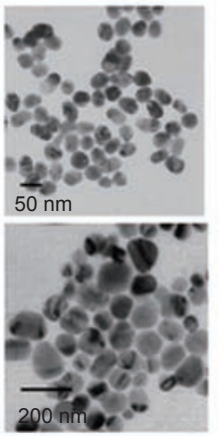

AgNPs100 (nm )

D

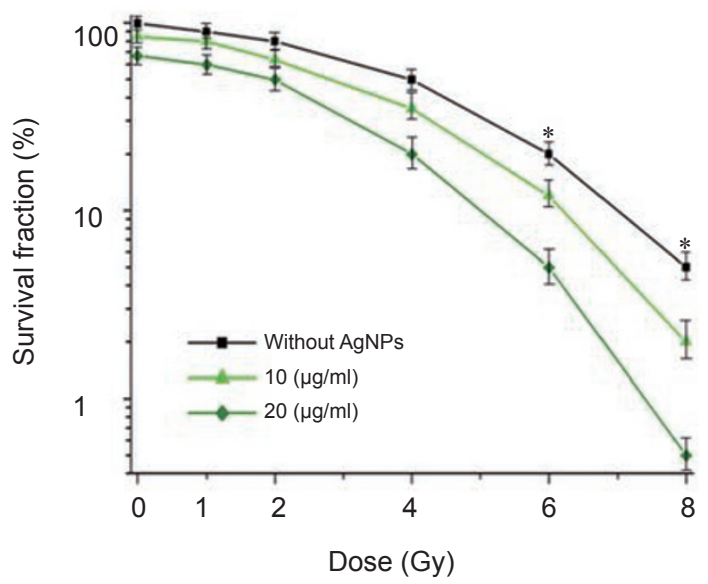

$\mathrm{F}$

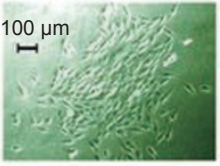

$a_{1}$

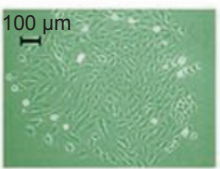

$b_{1}$

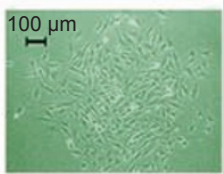

$\mathrm{C}_{1}$

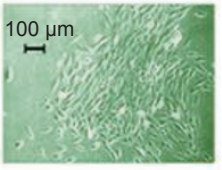

$\mathrm{a}_{2}$

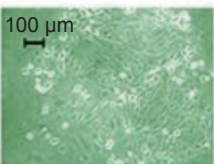

$\mathrm{b}_{2}$

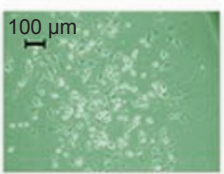

$\mathrm{C}_{2}$

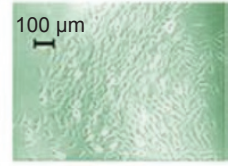

$a_{3}$

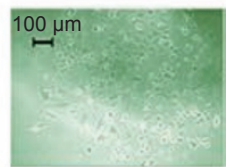

$b_{3}$

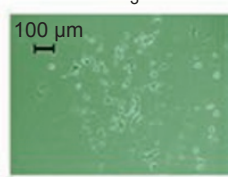

$\mathrm{C}_{3}$ 
bination of IR and $\mathrm{COOH}$ - or $\mathrm{NH}_{2}$-silane-MNPs treatment further reduced cell survival (Figure 1C). However, the inspection of the quantified results across different doses of IR indicated that $\mathrm{COOH}-$ or $\mathrm{NH}_{2}$-silane-MNPs caused a similar level of reduction of cell survival with or without IR (Figure 1C). Thus, these nanoparticles did not appear to increase cell radiation sensitivity per se; rather, they likely further increased cell killing by IR as a result of their general cytotoxicity toward these cells. Moreover, since MNPs with different surface coatings showed distinct effects on cell survival, we conclude that surface coating molecule could critically affect MNPcell interactions and consequently influence cellular outcomes. We also performed similar experiments in U251 and SHG-44 cells and found similar results to C6 cells (data not shown).

We next treated SHG-44 glioma cells with our FBSmodified AuNPs, and found that our AuNPs had little overall effect on cell survival, nor did they affect cell survival across different doses of IR. Similar results were obtained with C6 and U251 cells. Thus, these AuNPs, ranging from 10 to $40 \mathrm{~nm}$, do not appear to influence cell radiation sensitivity, at least under our experimental conditions. Our results are in contrast with previous reports showing that certain AuNPs coated with PEG or amino acids could enhance radiation-induced cytotoxicity in mice colorectal adenocarcinoma and breast cancer cells $[9,10]$. While the reason for this discrepancy is currently unknown, it raises an interesting possibility that different coatings of the AuNPs used in these studies may be responsible for the different outcomes observed.

Treating glioma cells with AgNPs led to dose-dependent cytotoxicity, with the smaller size particles (20 and $50 \mathrm{~nm}$ ) being most cytotoxic (Supplementary information, Figure S5); and these results are consistent with previous studies using other cell lines [2, 7]. To determine whether AgNPs affect cell radiation sensitivity, for each size of AgNPs, we chose a dose that when present alone only minimally affects cell survival, which is at about
$1 / 10$ to $1 / 5$ of the concentration required to kill $50 \%$ of the cells. Interestingly, we found that at such relatively harmless doses, both 20 and $50 \mathrm{~nm}$ AgNPs significantly enhanced radiation sensitivity of U251 cells, with $20 \mathrm{~nm}$ particles performing better than $50 \mathrm{~nm}$ ones (Figure 1D and $1 \mathrm{E}$ ), while the effect of $100 \mathrm{~nm} \mathrm{AgNPs} \mathrm{was} \mathrm{much}$ weaker. A similar particle size-dependent radiation sensitization effect was also observed for C6 and SHG-44 cells. Taken together, our results demonstrate that $20 \mathrm{~nm}$ and $50 \mathrm{~nm}$ AgNPs could function to enhance radiationinduced killing of glioma cells.

We hypothesize that the mechanism of radio-sensitization by AgNPs might be related to the release of $\mathrm{Ag}^{+}$cation from the silver nanostructures inside cells (Supplementary information, Figure S6). $\mathrm{Ag}^{+}$cation has the ability to capture electron and thus functions as an oxidative agent, which could further reduce ATP content of the cell and increase production of reactive oxygen species [8]. Indeed, treating cells of an established colony with $\mathrm{Ag}^{+}$ cation led to rapid cytotoxicity (Figure 1F), and further IR completely killed the cells within the colony. Consistent with our radio-sensitization results, treating cell colonies with $20 \mathrm{~nm}$ AgNPs led to some cytotoxicity on its own while it greatly enhanced further cell killing by IR (Figure 1F). The fact that the radio-sensitization effect of AgNPs decreases upon the increase of particle size is consistent with the $\mathrm{Ag}^{+}$cation release model, as smaller size AgNPs have a better ability to release silver ion.

Based on our results, we suggest that cellular effects of nanoparticles could be mediated by two distinct mechanisms that are contributed by either the particle itself or the nature of its surface coating. Among the MNPs, those coated with DMSA- and PEG-silane are relatively nontoxic, while MNPs coated by $\mathrm{COOH}-$ and $\mathrm{NH}_{2}$-silane show obvious toxicity to cells. As a result, combination of the cytotoxic MNPs with IR led to more cell killing than IR alone, but the effect is largely additive and these MNPs do not appear to increase cell radiosensitivity per se. While AuNPs showed little effect in our experiments,

Figure 1 Two types of nanoscale systems designed to investigate the effect on IR. (A) Monodisperse MNPs at the diameter of $12 \mathrm{~nm}$ coated with DMSA, and silanes bearing various functional groups including $\mathrm{NH}_{2}$, short-chain $\mathrm{PEG}$ and $\mathrm{COOH}$. AuNPs at 10, 20 and $40 \mathrm{~nm}$. AgNPs at 20, 50 and $100 \mathrm{~nm}$. (B) MNPs coated with DMSA and PEG-silane (0.1 mg/ml) showed little toxicity on $\mathrm{C} 6$ cells and did not affect cell killing by IR, while MNPs coated with $\mathrm{COOH}$-silane and $\mathrm{NH}_{2}-\mathrm{Silane}(0.1 \mathrm{mg} / \mathrm{ml})$ reduced cell survival. (C) At every dose of $\mathrm{X}$ ray, from left to right the data of control, MNPs-DMSA, $-\mathrm{PEG},-\mathrm{COOH}$ and $-\mathrm{NH}_{2}$ groups. (D and E) Dose- and size-dependent effects of AgNPs upon IR. The data represent the mean \pm SD of three independent experiments. $* P<0.05$ was considered statistically significant. (D) The survival curves of U251 cells treated with 10 and $20 \mu \mathrm{g} / \mathrm{ml} 20 \mathrm{~nm}$ AgNPs. (E) The survival curve of U251 cells treated with $20 \mathrm{~nm}(20 \mu \mathrm{g} / \mathrm{ml}), 50 \mathrm{~nm}(50 \mu \mathrm{g} / \mathrm{ml}) \mathrm{and} 100 \mathrm{~nm}(100$ $\mu \mathrm{g} / \mathrm{ml})$ AgNPs. (F) $\left(a_{1}\right),\left(b_{1}\right)$ and $\left(c_{1}\right)$ were photos of C6 colonies; $\left(b_{2}\right)$ was colony $\left(b_{1}\right)$ after $24 \mathrm{~h}$ of $20 \mathrm{~nm} \mathrm{AgNPs}(20 \mu g / m l),\left(c_{2}\right)$ was $\left(c_{1}\right)$ after $24 \mathrm{~h}$ of $\mathrm{Ag}^{+}(0.1 \mu \mathrm{g} / \mathrm{ml})$, and $\left(a_{2}\right)$ was $\left(a_{1}\right)$ with mock treatment. Colonies in $\left(a_{2}\right),\left(b_{2}\right)$ and $\left(c_{2}\right)$ then received $3 \mathrm{~Gy}$ of IR and were photographed after another $24 \mathrm{~h}$, shown in $\left(\mathrm{a}_{3}\right),\left(\mathrm{b}_{3}\right)$ and $\left(\mathrm{c}_{3}\right)$. 
AgNPs of particular sizes $(20 \mathrm{~nm}$ and $50 \mathrm{~nm})$ significantly sensitized glioma cells to IR. The radio-sensitization effect of AgNPs suggests that intracellular nanostructures may interact with the cellular DNA repair system, and has important implications in the design of nanotechnology-based radiosensitizers for improving the outcomes of cancer radiotherapy.

\section{Acknowledgments}

Financial support was provided by National Basic Research Program of China (973 Program) (No.2006CB933206) and the Scientific Research Foundation of Graduate School of Southeast University.

Ruizhi $\mathrm{Xu}^{1}$, Jun $\mathrm{Ma}^{2,3}$, Xinchen Sun ${ }^{4, *}$, Zhongping Chen $^{1}$, Xiaoli Jiang ${ }^{1}$, Zhirui Guo ${ }^{4}$, Lan Huang ${ }^{1}$, Yang $\mathrm{Li}^{1}$, Meng Wang ${ }^{1}$, Changling Wang ${ }^{1}$, Jiwei Liu ${ }^{1}, \mathrm{Xu}$ Fan $^{1}$, Jiayu $\mathrm{Gu}^{1}$, Xi Chen ${ }^{1}$, Yu Zhang ${ }^{1}$, Ning Gu, ${ }^{1, *}$

${ }^{I}$ School of Biological Science and Medical Engineering, $\mathrm{Ji}$ angsu Laboratory for Biomaterials and Devices, State Key Laboratory of BioElectronics, Southeast University, Nanjing 210096, China; ${ }^{2}$ Clinical college of Southeast University, Nanjing 210019, China; ${ }^{3}$ Tumor Center of Zhongda Hospital, Southeast University, Nanjing 210019, China; ${ }^{4}$ The Institute of Cardiovascular Disease, Division of Medicine, the First Affiliated Hospital of Nanjing Medical University, Nanjing 210029,

China

*These two authors contributed equally to this work.

Correspondence: Ning Gu

E-mail: guning@seu.edu.cn

\section{References}

1 Chithrani BD, Ghazani AA, Chan WCW. Determining the size and shape dependence of gold nanoparticle uptake into mammalian cells. Nano Lett 2006; 6:662-668.

2 Chung YC, Chen IH, Chen CJ. The surface modification of silver nanoparticles by phosphoryl disulfides for improved biocompatibility and intracellular uptake. Biomaterials 2008; 29:1807-1816.

3 Verma A, Uzun O, Hu YH, et al. Surface-structure-regulated cell-membrane penetration by monolayer-protected nanoparticles. Nat Mater 2008; 7:588-595.

4 Cedervall $\mathrm{T}$, Lynch I, Lindman S, et al. Understanding the nanoparticle-protein corona using methods to quantify exchange rates and affinities of proteins for nanoparticles. Proc Natl Acad Sci USA 2007; 104:2050-2055.

5 Lundqvist M, Stigler J, Elia G, et al. Nanoparticle size and surface properties determine the protein corona with possible implications for biological impacts. Proc Natl Acad Sci USA 2008; 105:14265-14270.

6 Pisanic TR, Blackwell JD, Shubayev VI, et al. Nanotoxicity of iron oxide nanoparticle internalization in growing neurons. Biomaterials 2007; 28:2572-2581.

7 Jiang W, Kim BYS, Rutka JT, et al. Nanoparticle-mediated cellular response is size-dependent. Nat. Nanotechnol 2008; 3:145-150.

8 AshaRani PV, Mun GLK, Hande MP, et al. Cytotoxicity and genotoxicity of silver nanoparticles in human cells. ACS Nano 2009; 3:279-290

9 Liu CJ, Wang CH, Chien CC, et al. Enhanced X-ray irradiation-induced cancer cell damage by gold nanoparticles treated by a new synthesis method of polyethylene glycol modification. Nanotechnology 2008; 19:1-5.

10 Kong T, Zeng J, Wang XP, et al. Enhancement of radiation cytotoxicity in breast-cancer cells by localized attachment of gold nanoparticles. Small 2008; 4:1537-1543.

(Supplementary information is linked to the online version of the paper on the Cell Research website.) 